The adequate transthoracic echocardiogram was possible in 18 (90\%) of potential organ donors. All examinations were performed before evaluation protocol confirmed brain death.

Results echocardiogram was normal in 13 (65\%) patients. One had a moderate mitral and tricuspid insufficiency. Minimal pericardial effusion was present in $4(20 \%)$ patients. Two had mild septal dyskinesia with normal left ventricular ejection fraction. Diffuse hypokinesis with ejection fraction of less than $55 \%$ was found in $5(37 \%)$ patients, in one of which it was less than $45 \%$. A total of 14 hearts $(70 \%)$ were harvested for transplantation, including the one with the poorest systolic function.

Conclusion mild left ventricular systolic dysfunction occurs frequently in children with brain death, but these hearts can still be considered for transplantation.

\section{LONG QT SYNDROME -A CASE REPORT}

Anupama Mallappa*, Detlev Rogahn. Royal Aberdeen Children's Hospital

\subsection{6/archdischild-2021-europaediatrics. 184}

Timothy syndrome is a rare genetic disorder characterized by an abnormally prolonged cardiac 'repolarization' time (long QT interval). This predisposes individuals to arrhythmias, cardiac arrest and sudden death.

Other features associated with this syndrome are dysmorphic facial features, webbing of fingers and/or toes (syndactyly); congenital heart defects, developmental delays and autism.

We want to report a case of Timothy syndrome, incidentally detected post induction for orchidopexy

Background TS is an extremely rare genetic disorder of the Ltype cardiac channel

Cav1.2 encoded by CACNA1C. The syndrome is characterized by multisystem abnormalities consisting of QT prolongation, congenital heart defects, syndactyly, facial dysmorphism, and neurological symptoms.

Case Report 8yr old boy was admitted to hospital for elective left orchidopexy, during induction developed 2degree AV block with T Talternans, maintained reasonable cardiac output throughout, QTc 504 msec. Past medical history an episode of syncope needing hospitalization. Currently being evaluated for autism.

Physical exam was normal. Holter, showed QTc prolongation with $\mathrm{T}$ alternans.

Genetic testing showed he is positive gene mutation CACNA1C. Parents have been counselled for the need for implantable defibrillator. $\mathrm{He}$ has been given external automated defibrillator in the meantime.

Currently on nadolol $40 \mathrm{mg} \mathrm{OD}$, parents are awaiting gene testing.

Discussion Classic timothy syndrome (TS) is a rare genetic disorder with dysfunction in multiple organ systems, clinically characterized by long QT syndrome and syndactyly. Timothy syndrome was first described in 1992. Classic TS is caused by a single missense mutation G406R of exon 8A of the Cav1.2 L-type calcium channel gene (CACNA1C) and is inherited in an autosomal dominant fashion, although it usually is the result of a de novo mutation. Patients with TS are prone to life threatening ventricular arrhythmias as a consequence of prolonged QT interval.
Since the affected gene is widely expressed in multiple adult and foetal tissues including gastrointestinal system, brain, lungs, immune system and testis, extracardiac manifestations are common.

The risk for life-threatening ventricular tachyarrhythmia is the limiting factor of TS. Since ventricular tachyarrhythmia is the leading cause of death in patients with TS, effective antiarrhythmic medication and an implantable cardioverter defibrillator are the mainstay of therapy Conclusions Timothy syndrome is a rare congenital arrhythmia disorder with dysfunction in multiple organ systems. Patients are at high risk for sudden death due to life threatening ventricular tachyarrhythmia. Implantation of an ICD at a very young age may be the best means to prevent sudden death.

\section{CORONARY ANEURYSM - A REALITY IN KAWASAKI DISEASE ASSOCIATED TO COVID 19}

Nitescu Gabriela Viorela*, Alexandru Paul Baciu, Carmen Gavrila, Cristina Raduta, Marian Alexe, Sorina Stuparu, Usurelu Diana, Cristina Olteanu, Evelina Cotora. ${ }^{1}$ Emergency Clinical Hospital for Children 'Grigore Alexandrescu',Bucharest Romania, University of Medicine and Pharmacy 'Carol Davila', Bucharest Romania; 'University of Medicine and Pharmacy 'Carol Davila' Bucharest

\subsection{6/archdischild-2021-europaediatrics. 185}

Introduction Kawasaki disease is an acute febrile vasculitis, primarly affecting children, that is epidemiologically and clinically closely linked to a viral infection (2). This entity can sometimes have severe forms. Numerous clinical studies published in the last two years have shown that infection with SARSCoV2 virus (COVID 19) in children is frequently associated with this entity, which can sometimes be severe.

We will present the case of a 9-month-old infant, without personal history, who was hospitalized for fever.

The onset of the disease was 14 days earlier, with high fever and rash. He was hospitalized in another clinic, where the diagnosis of Covid 19-associated Kawasaki Disease was established (the criteria were as follows: fever for longer than 5 days, papulo-erythematous rash on the chest and limbs, changes in the oral and lip mucosa, palmo-plantar edema, bilateral conjunctivitis, Ig $\mathrm{G}$ anti SARS $\mathrm{CoV} 2=4.52 \mathrm{u} / \mathrm{ml}$ ).

He received treatment with Gamma globulin $2 \mathrm{~g} / \mathrm{kg}$ in single dose, corticosteroid therapy, Aspirin.

The evolution was initially favorable. But after 7 days, high fever reappeared. Then, he is hospitalized in our clinic. At admission he had high fever, hyperemic lips, bilateral conjunctivitis, no manifestations of cardiac dysfunction, leukocytosis: $26,700 / \mathrm{mmc}$, marked inflammatory syndrome $(\mathrm{ESR}=130 \mathrm{~mm} /$ h, C-reactive protein $=8.8 \mathrm{mg} / \mathrm{dl}$ ). Cardiac ultrasound detects an aneurysm of the coronary arteries $(\mathrm{RCA}=3.5 \mathrm{~mm}, \mathrm{Z}$ score $=6, \mathrm{LCA}=2.5 \mathrm{~mm}, \mathrm{Z}$ score $=1.43$ ).

The diagnosis of Kawasaki disease refractory to immunoglobulin treatment has been established, resuming administration in a dose of $400 \mathrm{mg} / \mathrm{kg}$ of body weight/day for 3 days.

Treatment was supplemented with intravenous methylprednisolone pulse therapy, aspirin and enoxaparin.(1) The evolution was favorable with the remission of clinical symptoms and inflammatory syndrome, but with the persistence of coronary dilation.

He was discharged from hospital with good general condition, normal inflammatory markers, changes in lipid profile (total cholesterol=306 mg/dl, total lipids=951 $\mathrm{mg} / \mathrm{dl}$, 